\title{
Modes de gestion et efficacité de conservation des reliques de forêts naturelles en zone tropicale sèche : cas du Département de Korhogo (Nord, Côte d'Ivoire)
}

\author{
Nina GUEULOU ${ }^{1,2^{*}}$, Brahima COULIBALY ${ }^{2}$, Noufou Doudjo OUATTARA ${ }^{1,3}$, \\ Anatole Kanga N'GUESSAN ${ }^{2}$, Assandé AHOBA ${ }^{2}$ et Adama BAKAYOKO ${ }^{1,3}$
}

\author{
${ }^{1}$ UFR des Sciences de la Nature (SN), Université Nangui Abrogoua, 02 BP 801 Abidjan 02, Côte d'Ivoire. \\ ${ }^{2}$ Station de Recherche Technologique, Centre National de Recherche Agronomique (CNRA), 08 BP 33 Abidjan \\ 08, Côte d'Ivoire. \\ ${ }^{3}$ Centre Suisse de Recherches Scientifiques en Côte d'Ivoire, 01 B.P. 1303 Abidjan 01, Côte d'Ivoire. \\ *Auteur correspondant ; E-mail : ninagueulou@gmail.com
}

\section{RESUME}

L'étude a été réalisée dans trois îlots de forêts naturelles caractérisées par trois différents modes de gestion dans les zones sèches de Côte d'Ivoire. Son objectif est d'évaluer la composition et la diversité floristique de ces reliques forestières afin de promouvoir le meilleur mode de gestion pour la conservation de ces forêts. L'échantillonnage de la végétation a été conduit dans les îlots forestiers de Lataha, de Dolékaha et de Korhogo. La collecte des données a été réalisée à l'aide de la méthode de relevé de surface complétée par des inventaires itinérants. Tous les individus de diamètre à hauteur de poitrine $(\mathrm{dbh}) \geq 5 \mathrm{~cm}$ ont été mesurés dans des placettes carrées de $25 \mathrm{~m}$ de côté. Les résultats révèlent une plus forte richesse spécifique sur le site de Lataha $(19,25 \pm 4,55)$ et de Korhogo $(17,72 \pm 3,28)$. Les valeurs des indices de Shannon indiquent que la forêt de Korhogo est la plus diversifiée $(2,47 \pm 0,28)$. Pour l'indice d'équitabilité de Piélou, la valeur la plus importante est similaire pour les sites de Korhogo et de Dolékaha $(0,84 \pm 0,05)$. Le site de Korhogo regorge encore la plus forte présence des espèces à valeur de conservation. La forêt de Korhogo qui bénéficie d'une cogestion comporte la meilleure composition et diversité floristique. De ce fait, des aménagements forestiers doivent de plus en plus associer les populations riveraines pour une gestion participative. Nos résultats peuvent être utiles pour élaborer des stratégies de conservation efficace des reliques forestières de toutes les zones phytogéographiques du pays et même ailleurs dans le monde.

(c) 2019 International Formulae Group. All rights reserved.

Mots clés: Forêts naturelles, zone sèche, modes de gestion, efficacité de conservation, Korhogo, Côte d'Ivoire.

\section{Management and effectiveness of conservation of natural forest relics in dry tropical zone: case of the Department of Korhogo (North, Côte d'Ivoire)}

\begin{abstract}
This study was conducted in three types of natural forest distinguished by three different management methods in dry areas of Côte d'Ivoire. Its aims at assessing the floristic and diversity composition of these forest relics in order to promote the best mode of management for the conservation of these forests. Botanical
\end{abstract}


sampling was conducted in the forest relics of Lataha, Dolékaha and Korhogo. Data collection was performed using surface survey method followed by the itinerant inventories. All individuals at a breast height diameter $(\mathrm{dbh}) \geq 5 \mathrm{~cm}$ were measured in $25 \mathrm{~m}$ square plots. The results reveal a higher species richness at the Lataha $(19.25 \pm 4.55)$ and Korhogo $(17.72 \pm 3.28)$ site. Shannon index values indicate that the Korhogo forest is the most diversified $(2.47 \pm 0.28)$. For Piélou's index, the highest value is similar for the Korhogo and Dolékaha sites $(0.84 \pm 0.05)$. The Korhogo site still has the highest presence of species with conservation value. This study showed that the Korhogo forest, which benefits from co-management, has the best floristic and diversity composition. As a result, natural forest developments must increasingly involve local populations for participative management. Our results can be useful in developing strategies for the effective conservation of forest relics in all phytogeographic zones of Côte d'Ivoire and globally.

(C) 2019 International Formulae Group. All rights reserved.

Keywords: Natural forests, dry zone, management methods, effectiveness of conservation, Korhogo, Côte d'Ivoire.

\section{INTRODUCTION}

La conservation de la biodiversité en Afrique repose en grande partie sur l'efficacité des systèmes d'aires protégées (Ervin, 2003). Les aires protégées sont des espaces géographiques clairement définis, reconnus, consacrés et gérés, par tout moyen efficace, juridique ou autre, afin d'assurer à long terme la conservation de la nature ainsi que les services écosystémiques et les valeurs culturelles qui lui sont associés (Dudley, 2008). Ces espaces généralement constitués par les parcs nationaux, les réserves naturelles et les forêts classées mettent la Côte d'Ivoire en tête des pays francophones d'Afrique de l'Ouest dans le domaine de la conservation de la nature (Chappe et al., 2003).

Dans les pays africains, cette conservation et protection de la biodiversité s'avère plus difficile à appliquer en dehors des aires protégées où les forêts sont facilement affectées par diverses activités anthropiques. En Côte d'Ivoire, cette situation est plus accentuée en zone soudanienne. Dans ces écosystèmes de savane, la végétation naturelle initiale s'est progressivement dégradée sous l'action des pressions agricoles, pastorales et des feux de brousse (Ouattara, 2001). Toutefois, même dans ces zones de grandes menaces de désertification, subsistent encore des reliques de forêts sacrées, de forêts classées et de forêts à vocations scientifiques qui sont aujourd'hui les seuls lieux de concentration de la phytodiversité (Koulibaly, 2000; Tiébré et al., 2016). Il se pose alors le problème de la bonne conservation de ces ressources forestières. Selon Poisson (2009), l'efficacité de conservation des forêts dépend de la qualité du mode de gestion. A cet effet, l'Union Internationale pour la Conservation de la Nature (UICN) reconnaît quatre grands types de gestion (Dudley, 2008). Chacun d'eux pouvant être associé à des objectifs de gestion très variés allant de la protection intégrale à l'utilisation durable des ressources forestières. Certains sites sont gérés par des gouvernements, d'autres par des particuliers, des organisations non gouvernementales, des sociétés, des structures de recherches, d'autres par des communautés locales. Quant à la gestion collaborative ou conjointe, l'autorité et la responsabilité de gestion sont partagés par divers acteurs.

Avec les travaux de Vroh et al. (2015) dans les zones humides de la Côte d'Ivoire, l'on se rend compte aujourd'hui que la conservation et la gestion responsable des forêts font partie des stratégies les plus rentables dans la conservation de la biodiversité. Dans les zones sèches du pays, peu d'études similaires ont été réalisées. La plupart des travaux scientifiques menés jusqu'à ce jour se sont focalisés sur la caractérisation floristique de ces formations végétales, l'inventaire des plantes utilitaires et les différentes méthodes de sylviculture et d'aménagement (Tiébré et al., 2016; Yéo et al., 2017 ; N'Guessan et al., 2018). Les travaux traitant des impacts des modes de gestion sur la composition et la diversité floristique des îlots forestiers des zones sèches sont quasi inexistants. Il est donc 
indispensable de détecter le mode de gestion le plus efficace afin de soutenir et de promouvoir des forêts plus résilientes face aux différentes perturbations qu'elles subissent. Cette étude concerne trois types de forêts naturelles distingués par trois différents modes de gestion : La forêt de Lataha protégée par un organisme public, celle de Dolékaha gérée par les populations locales et celle de Korhogo associant ces deux modes de gestion. L'objectif principal a été de comparer l'impact de ces modes de gestion sur l'efficacité de conservation de ces reliques forestières en zone sèche. De façon spécifique, il s'est agi de déterminer la richesse, la composition et la diversité floristique de chacun des ilots forestiers subissant différentes perturbations. Cette recherche s'est fondée sur l'hypothèse selon laquelle différents modes de gestion influencent la diversité et la composition floristique des forêts en zone sèche.

\section{METHODES D'ETUDE \\ Zone d'étude}

Cette étude a été réalisée dans le Département de Korhogo, dans la Région du Poro, au Nord de la Côte d'Ivoire. La zone d'étude est située entre $9^{\circ} 20^{\prime}$ et $9^{\circ} 40^{\prime}$ de latitude Nord et entre $5^{\circ} 95^{\prime}$ et $5^{\circ} 75^{\prime}$ de longitude Ouest, dans le secteur sub-soudanais $\mathrm{du}$ domaine soudanais (Guillaumet et Adjanohoun, 1971). Elle couvre une superficie de 12640,4 $\mathrm{km}^{2}$, avec une population de 536851 habitants (RGPH, 2014). La température moyenne annuelle oscille autour de $27^{\circ} \mathrm{C}$. La pluviométrie est de $1200 \mathrm{~mm} / \mathrm{an}$ avec une très forte variabilité interannuelle. La végétation se caractérise essentiellement par des formations de savanes (savanes herbeuses, arbustives ou arborées), des ilots de forêts galeries, de forêts denses sèches et de forêts claires. Ces formations végétales sont soumises à un climat de type soudanais, qui présente une saison pluvieuse courte et une longue saison sèche ( 9 mois) avec l'harmattan (vent sec) qui dure 3 à 5 mois. Les sols sont essentiellement de type ferrugineux et ferralitique (Koné, 2004) (Figure 1).

\section{Sites inventoriés}

L'inventaire floristique a été réalisé dans trois îlots de forêts naturelles distingués par trois différents modes de gestion. Ce sont : la forêt de Lataha protégée par une structure de recherche, le Centre National de Recherche Agronomique (CNRA). La forêt sacrée de Dolékaha gérée par la population locale (autorités coutumières du village Dolékaha) et enfin la forêt classée de Korhogo qui associe ces deux modes de gestion (la Société de Développement des Forêts (SODEFOR) et la population du village Sehèlè). Ces trois sites sont soumis à une protection intégrale, mais compte tenu de leur localisation à proximité des villages, ils sont victimes des agressions et activités illégales.

Dans la forêt de Lataha, il a été uniquement constaté la récolte de plantes médicinales. Ce site situé en bordure d'un barrage hydroagricole est une vielle jachère d'environ 15 ha dont les premières mesures de protection n'ont été prises qu'en 1988 (30 ans de mise en défens). Environ 70\% de la surface forestière se caractérise par l'existence d'une canopée ouverte.

La forêt sacrée de Dolékaha est une forêt dense sèche de plus de 150 ans et s'étend sur une superficie d'environ 5 ha. L'intensité de la pression anthropique y est plus élevée par rapport aux autres forêts. En effet, elle est soumise à la dent de bétail, à la chasse et au prélèvement de bois mort pour le chauffage.

$\mathrm{La}$ formation naturelle de la forêt classée de Korhogo est plus ou moins intacte avec de faible pression pastorale. Des contrôles réguliers y sont effectués par la SODEFOR. Un comité de surveillance composé de cinq riverains aide les agents à lutter efficacement contre les infiltrations illégales, les feux de brousse, les pâturages clandestins, les exploitations frauduleuses des produits forestiers (SODEFOR, 2018). C'est la forêt sacrée du village de Séhèlè qui s'est vue classée par le gouvernement en 1953. C'est une portion enclavée de forêt galerie inondée par des cours d'eau.

\section{Collecte des données}

L'étude a été réalisée durant les mois d'octobre et de novembre 2018. Les données ont été acquises à l'aide de deux méthodes 
complémentaires d'inventaire : le relevé de surface et le relevé itinérant (Aubreville, 1959). Le relevé de surface a consisté à installer des placettes de $25 \mathrm{~m}$ x $25 \mathrm{~m}(625$ $\mathrm{m}^{2}$ ) dans chacun des trois sites forestiers et à y inventorier les espèces végétales ciblées dans cette étude : les ligneux de diamètre à hauteur de poitrine (dbh) supérieure ou égale à cinq centimètres (Marthews et al., 2012). Au total 16 parcelles ont étés inventoriées à Lataha, 14 parcelles à Dolékaha et 11 parcelles à Korhogo. Des relevés itinérants ont été réalisés. Ils ont consisté à recenser tous les spécimens de chaque nouvelle espèce rencontrée, qui n'ont pas été inventoriés dans les relevés de surface en vue de compléter la liste floristique de la zone d'étude. Ils se sont effectués au gré de promenades à travers la végétation. Toutes les espèces ont été identifiées à l'herbier du Centre Suisse de Recherches Scientifiques en Côte d'Ivoire (CSRS). La nomenclature adoptée est celle de la classification phylogénétique (APG IV, 2016). La désignation des types morphologiques, biologiques et phytogéographiques a été réalisée en se référant aux travaux de Aké-Assi $(2001,2002)$ et de Chatelain et al. (2011).

\section{Analyse des données \\ Richesse floristique et composition floristique}

$\mathrm{La}$ richesse floristique d'un milieu désigne la liste de toutes les espèces végétales rencontrées dans les limites de ce milieu (Aké-Assi, 1984). Elle est généralement évaluée au niveau spécifique, générique et de la famille. L'analyse de la composition floristique a consisté à relever pour chaque espèce identifiée, le type biologique et l'affinité chorologique. Ces paramètres donnent des renseignements sur la stabilité et la dynamique de la forêt et peuvent servir d'indicateurs de l'intensité des perturbations passées de l'écosystème (Bakayoko, 2005). Dans cette étude, les types biologiques ont été définis selon les travaux de Aké-Assi (2001, 2002) et les affinités chorologiques selon les travaux de Chatelain et al. (2011). La terminologie utilisée est celle de Raunkiaer (1934) adaptée par Aubreville (1959). Pour chaque type de forêt, la valeur de conservation de la biodiversité a été évaluée à travers la détermination des espèces à statut particulier. $\mathrm{Ce}$ sont les espèces rares, menacées de disparition (ou d'extinction) ou encore endémiques (Adou et N'Guessan, 2005). Dans cette étude, le statut des espèces inventoriées a été déterminé selon la liste rouge de l'IUCN (2018) et celle des espèces menacées de AkéAssi (1998).

\section{Indices de diversité}

Indice de Shannon-Weaver ( $H^{\prime}$ )

L'indice de Shannon mesure la composition en espèces d'un peuplement en tenant compte de la richesse spécifique et de leur abondance relative (Felfili et al., 2004). Dans une communauté de $\mathrm{S}$ espèces, l'indice de Shannon-Weaver (H') se calcule selon la formule suivante :

$$
\mathrm{H}^{\prime}=-\sum_{\mathrm{i}=1}^{\mathrm{S}}\left[(\mathrm{Pi}) \log _{2}(\mathrm{Pi})\right](1) \text { avec } P \mathrm{i}=
$$
$\mathrm{ni} / \mathrm{Ni}$

Dans cette formule, $\mathrm{H}^{\prime}$ désigne l'indice de diversité de Shannon, ni le nombre d'individus d'une espèce $\mathrm{i}$ avec $\mathrm{i}$ allant de 1 à $\mathrm{S}$ (nombre total d'espèces), $\mathrm{N}$ le nombre total d'individus de toutes les espèces confondues dans le milieu, Pi la proportion relative du nombre d'individus de l'espèce $\mathrm{i}$ et $\log _{2}$ le logarithme en base 2. Les valeurs de cet indice varient entre 0 et $\log _{2} \mathrm{~S}$ qui est la diversité maximale ( $\mathrm{S}$ étant le nombre total d'espèces dans le milieu). Lorsque le peuplement est dominé par une seule espèce, il tend vers 0 , tandis que pour une flore comportant un nombre élevé d'espèces, il tend vers $\log _{2} \mathrm{~S}$.

\section{Indice d'équitabilité de Piélou (J')}

Cet indice décrit la répartition des effectifs des individus entre les différentes espèces d'un peuplement (Djègo et al., 2012). Il est déduit de celui de Shannon H' selon l'équation suivante :

$$
\mathrm{J}^{\prime}=\mathrm{H}^{\prime} / \log _{2} \mathrm{~S}(2)
$$

Avec J'_l'indice d'équitabilité de Piélou, H'_l'indice de diversité de Shannon, $\log _{2}$ le logarithme en base 2 et $S \_l a$ richesse spécifique de la parcelle ou du biotope considéré. Il varie de 0 à 1 . Si J' est faible, on conclut qu'il y a dominance des individus d'une espèce par rapport aux autres dans le milieu. Mais lorsqu'il est élevé, cela traduit une distribution régulière des individus entre 
les espèces du milieu. Dans ce cas le milieu en question est dit équilibré.

\section{$\beta$-diversité ou similarité floristique}

Pour vérifier la ressemblance ou la dissemblance entre nos différentes forêts, nous nous sommes servis du coefficient de similitude de Sørensen (1948) dont l'expression mathématique est :

$$
\operatorname{Cs}(\mathrm{s})=100 \times(2 \mathrm{c} /(\mathrm{a}+\mathrm{b}))(3)
$$

Avec a_ nombre d'espèces du milieu A, b_nombre d'espèces du milieu $B$ et c_nombre d'espèces communes aux deux milieux écologiques. Les valeurs de Cs varient entre 0 et $100 \%$. Plus les listes ont des espèces en commun, plus Cs tend vers $100 \%$. Plus les deux listes floristiques sont dissemblables, plus la valeur de Cs tend vers 0 .

\section{Analyses statistiques}

Le logiciel Past 2.16 a servi à calculer les indices de Shannon et d'équitabilité de Piélou. Il a également permis de réaliser la matrice de similarité de Sorensen. L'analyse de variance (ANOVA) à un facteur a été utilisée pour comparer les moyennes des paramètres tels que la richesse, la composition floristiques ainsi que les indices de diversité des différents biotopes prospectés. Le but de l'analyse est d'établir si les moyennes des valeurs mesurées dans différents groupes, sont significativement différentes. Elle repose sur trois principes : l'indépendance des observations, la normalité de la distribution et l'homogénéité des variances (l'homoscédasticité). La normalité est vérifiée par le test de Shapiro-Wilk et l'homogénéité des variances est vérifiée par le test de Battlet. Le niveau de significativité choisi pour ces analyses, est de 5\% ( $p$-value $=0,05)$. Lorsque la différence est significative, le test post ANOVA de Tukey a été réalisé pour classer et distinguer lesquels des îlots forestiers sont différents. Lorsque les conditions de l'ANOVA n'étaient pas respectées, le test non paramétrique de Kruskal-Wallis a été effectué. Ces tests statistiques ont été réalisés à l'aide du Package Rcommander du logiciel $\mathrm{R}$ Studio.

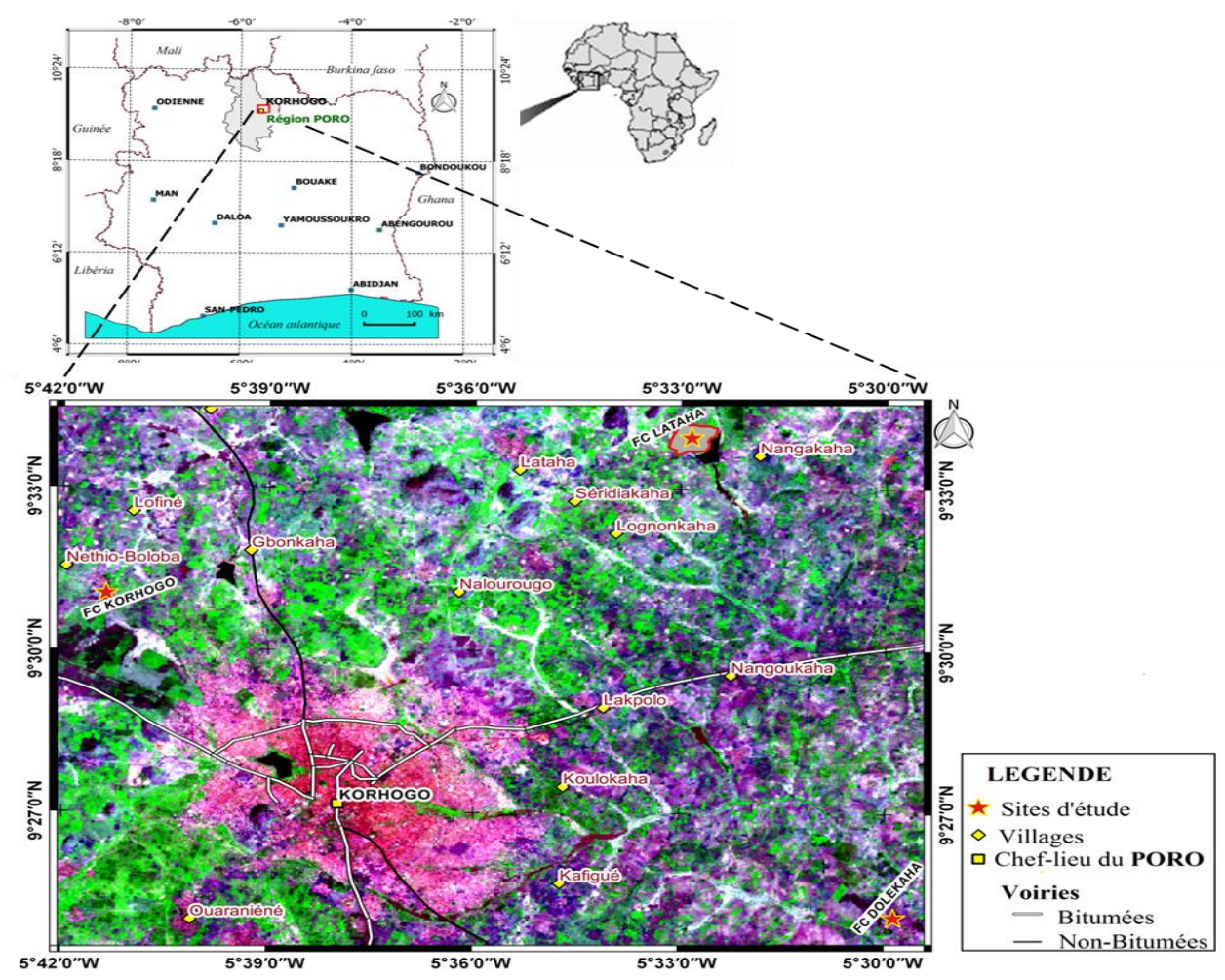

Figure 1 : Localisation des sites d'études (Landsat OLI du 22 / 02 / 2018). 


\section{RESULTATS}

\section{Richesse spécifique et composition floristique des biotopes}

$\mathrm{La}$ richesse floristique varie d'un fragment forestier à un autre. La forêt de Lataha possède la plus grande richesse floristique avec 74 espèces reparties en 68 genres et 26 familles. Elle est suivie de celle de Korhogo avec 65 espèces, 59 genres et 26 familles. La forêt sacrée de Dolékaha est la moins riche avec 49 espèces, 46 genres et 20 familles. L'analyse statistique a montré qu'il existe effectivement une différence significative $(F=6.212 ; P=0,0047)$ entre ces trois reliques forestières. Cette différence est plus élevée entre la forêt de Lataha et celle de Dolékaha (Tableau 1). A cet effet, l'allure des courbes aires-espèces traduit également la richesse floristique existante dans ces ilots de forêts, de sorte que la totalité des espèces n'a pas encore été inventoriée. Elle montre aussi qu'il existe une différence significative de cette richesse spécifique entre la forêt de Lataha et celle de Dolékaha, tandis que celle de Lataha et de Korhogo sont floristiquement proches (Figure 2).

Les familles botaniques les mieux représentées dans les différents biotopes sont les Fabaceae. Elles sont les plus rencontrées dans les trois formations forestières. Elles sont suivies de la famille des Rubiaceae dans les forêts de Lataha et de Korhogo, puis des Apocynaceae dans la forêt de Dolékaha (Tableau 1).

\section{Types biologiques et chorologiques}

Les microphanérophytes et les mésophanérophytes constituent les types biologiques majoritaires et les nanophanérophytes les types minoritaires au sein des trois biotopes (Figure 3). La proportion la plus élevée de microphanérophytes est observée dans la forêt de Lataha $(69,58 \%)$ et la plus faible à Dolékaha $(47,41 \%)$. Pour les mésophanérophytes, on observe la plus forte proportion sur le site de Korhogo (33,74\%) et la plus faible sur le site de Lataha $(25,10 \%)$. Les lianes microphanérophytes et mésophanérophytes prédominent dans les reliques forestières de Dolékaha (respectivement, $12,12 \%$ et $4,17 \%$ ) et de
Korhogo (respectivement, $12,11 \%$ et $2,03 \%$ ). Dans la forêt de Lataha on note une faible proportion des lianes microphanérophytes $(3,99 \%)$ et une absence des lianes mésophanérophytes. La forêt sacrée de Dolékaha détient la proportion la plus élevée de mégaphanérophytes $(4,97 \%)$ tandis qu'on observe une absence de ces ligneux sur le site de Lataha.

La proportion des espèces soudanoguinéennes (GC-SZ) est plus importante dans les forêts de Dolékaha $(69,68 \%)$ et de Korhogo $(73,04 \%)$ alors qu'elles sont moins importantes sur le site de Lataha (46,97\%). Sur ce dernier, prédominent les espèces soudaniennes (SZ) $(47,15 \%)$ avec une faible représentativité à Dolékaha $(4,57 \%)$. A l'inverse, la forêt de Dolékaha comporte le plus d'espèces guinéennes (GC) avec une proportion de $15,50 \%$ et la plus faible proportion pour le site de Lataha $(0,68 \%)$ et de Korhogo $(7,24 \%)$. On note également la présence des espèces endémiques ouestafricaines (GWC) à Korhogo (2,03\%) alors qu'elles sont pratiquement absentes des relevés de Lataha et de Dolékaha. Les proportions les plus élevées et les plus faibles d'espèces introduites ont été signalés successivement à Dolékaha (10,5\%), à Lataha $(5,06 \%)$ et Korhogo $(2,71 \%)$ (Figure 4$)$.

\section{Espèces à valeur de conservation}

La flore de l'ensemble des trois îlots forestiers comprend 12 espèces à statut particulier. Dix de ces espèces sont sur la liste rouge de l'UICN (2018) et six espèces sur la liste des espèces menacées de Aké-Assi (1998). Hormis Syzygium guineense (Engl.) F. White et Voacanga thouarsii var obtusa Roem. ex Schultes toutes les espèces se trouvant sur la liste de Aké-Assi figurent sur la liste rouge de l'UICN. Elles se répartissent en 10 espèces dites vulnérables, une espèce en danger d'extinction et une espèce quasi menacée. Le site de Lataha possède sept espèces dont six vulnérables et une en danger d'extinction. La forêt de Dolékaha contient six espèces vulnérables avec l'espèce Milicia excelsa (Welw.) C.C. Berg qui est aussi considérée par l'IUCN (2018) comme une espèce quasi menacée. Le site de Korhogo enregistre également neuf espèces à valeur de 
conservation, tous considérées comme des espèces vulnérables (Tableau 2).

\section{Analyse des indices de diversité}

Le test de comparaison des indices de Shannon montre que ces trois biotopes présentent des différences significatives $\left(X^{2}=\right.$ $1,66 ; P=0,435)$. La forêt de Korhogo est la

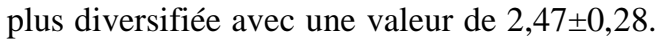
On note une valeur de 2, $36 \pm 0,58$ pour le site de Lataha. La forêt de Dolékaha possède la plus faible valeur de cet indice $(2,29 \pm 0,26)$ (Tableau 3). Pour l'indice d'équitabilité de Piélou, les valeurs les plus importantes, s'observent à Korhogo et Dolékaha (respectivement $0,84 \pm 0,05$ et $0,84 \pm 0,07$ ) . Lataha possède la plus faible valeur $(0,79 \pm$ $0,15)$. L'analyse statistique montre qu'il existe également une différence significative $\left(\mathrm{X}^{2}=\right.$ 6,26; $\mathrm{P}=0,043)$ entre les trois biotopes. Cette différence se perçoit entre le site de Lataha et les deux autres sites (Korhogo et Dolékaha) (Figure 5).

\section{Similarité floristique entre les îlots forestiers ( $\beta$-diversité)}

Le degré de ressemblance entre les trois formations naturelles est indiqué au Tableau 4. La plus forte ressemblance floristique est observée entre la forêt de Korhogo et celle de Lataha qui partagent $52 \%$ d'espèces. Les sites de Korhogo et de Dolékaha partagent $50 \%$ de ressemblance. La plus faible similarité s'observe entre la forêt de Lataha et celle de Dolékaha avec 44\% des espèces communes.

Tableau 1 : Richesse floristique des reliques forestières.

\begin{tabular}{|c|c|c|c|c|c|c|}
\hline \multirow[t]{2}{*}{ Sites } & \multirow{2}{*}{$\begin{array}{l}\text { Nombre de } \\
\text { placettes }\end{array}$} & \multirow{2}{*}{$\begin{array}{l}\text { Nombre } \\
\text { d'espèces }\end{array}$} & \multirow{2}{*}{$\begin{array}{l}\text { Nombre de } \\
\text { genres }\end{array}$} & \multicolumn{2}{|c|}{ Familles } & \multirow{2}{*}{$\begin{array}{l}\text { Richesse } \\
\text { Spécifique } \\
\text { moyenne }\end{array}$} \\
\hline & & & & Nombre & $\begin{array}{c}\text { Familles } \\
\text { dominantes }\end{array}$ & \\
\hline $\begin{array}{l}\text { Forêt de } \\
\text { Lataha }\end{array}$ & 16 & 74 & 68 & 26 & $\begin{array}{l}\text { Fabaceae et } \\
\text { Rubiaceae }\end{array}$ & $19,25 \pm 4,55 \mathrm{a}$ \\
\hline $\begin{array}{l}\text { Forêt de } \\
\text { Dolékaha }\end{array}$ & 14 & 49 & 46 & 20 & $\begin{array}{l}\text { Fabaceae et } \\
\text { Apocynaceae }\end{array}$ & $14,57 \pm 2,65 b$ \\
\hline $\begin{array}{l}\text { Forêt de } \\
\text { Korhogo }\end{array}$ & 11 & 65 & 59 & 26 & $\begin{array}{l}\text { Fabaceae et } \\
\text { Rubiaceae }\end{array}$ & $17,72 \pm 3,28 \mathrm{ab}$ \\
\hline$F$ & - & - & - & - & - & 6,212 \\
\hline$P$ & - & - & - & - & - & 0,0047 \\
\hline
\end{tabular}

Les valeurs portant des lettres différentes sont significativement différentes au seuil de 5\%.

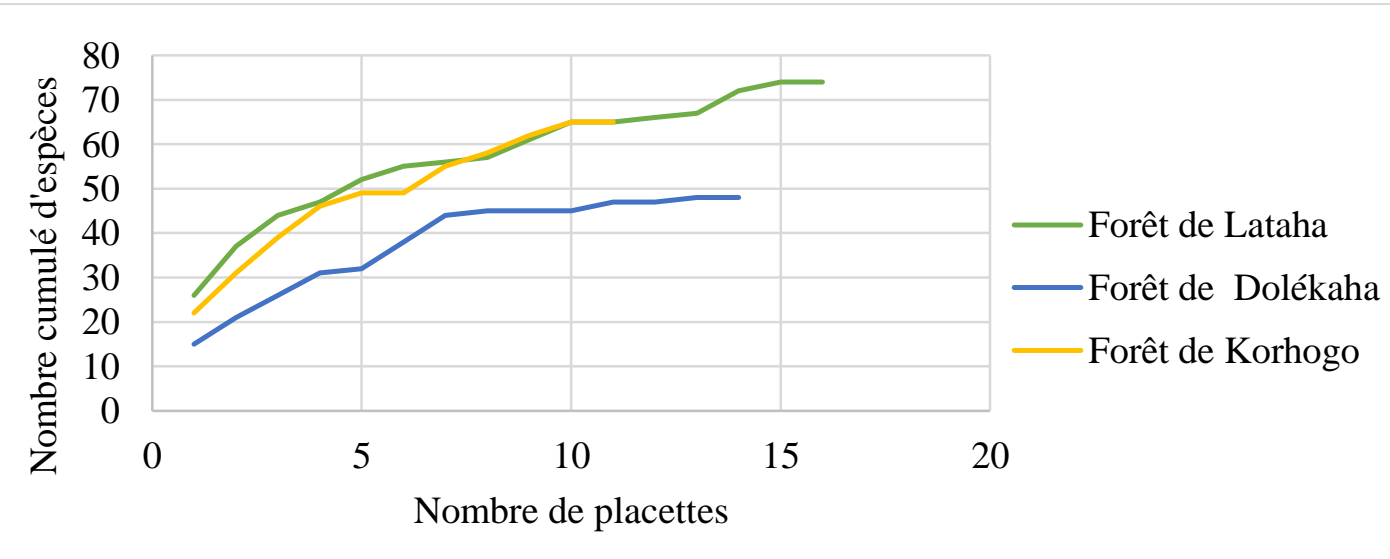

Figure 2 : Courbes aires-espèces des espèces inventoriées dans les îlots forestiers. 


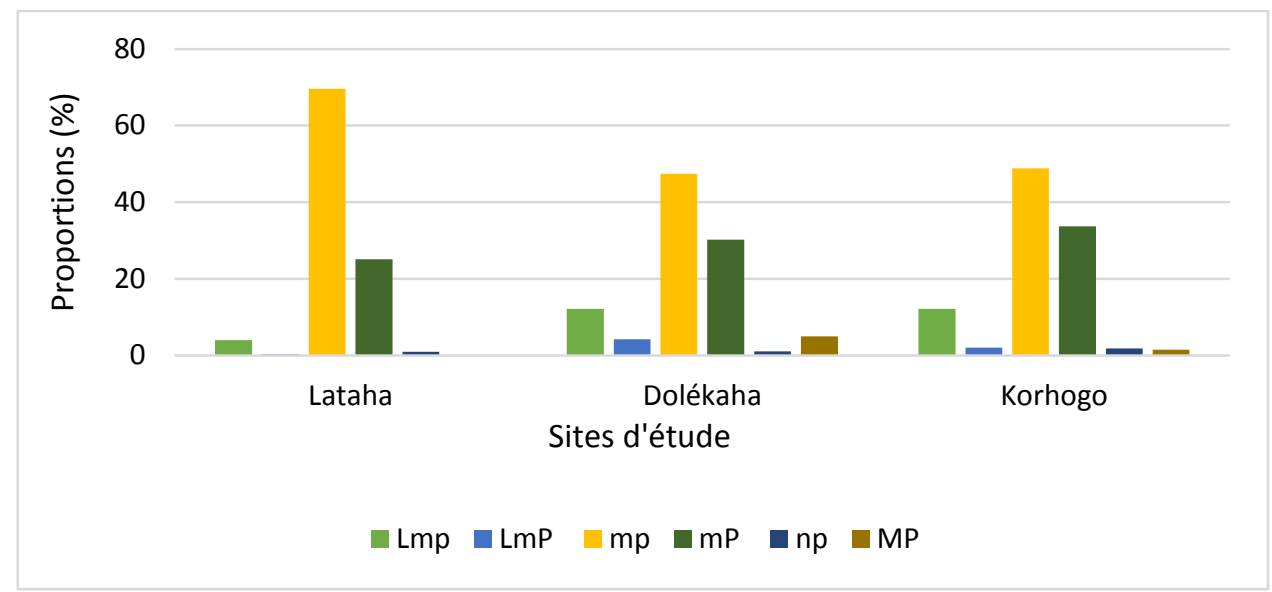

Figure 3 : Proportion des types biologiques en fonction des sites inventoriés.

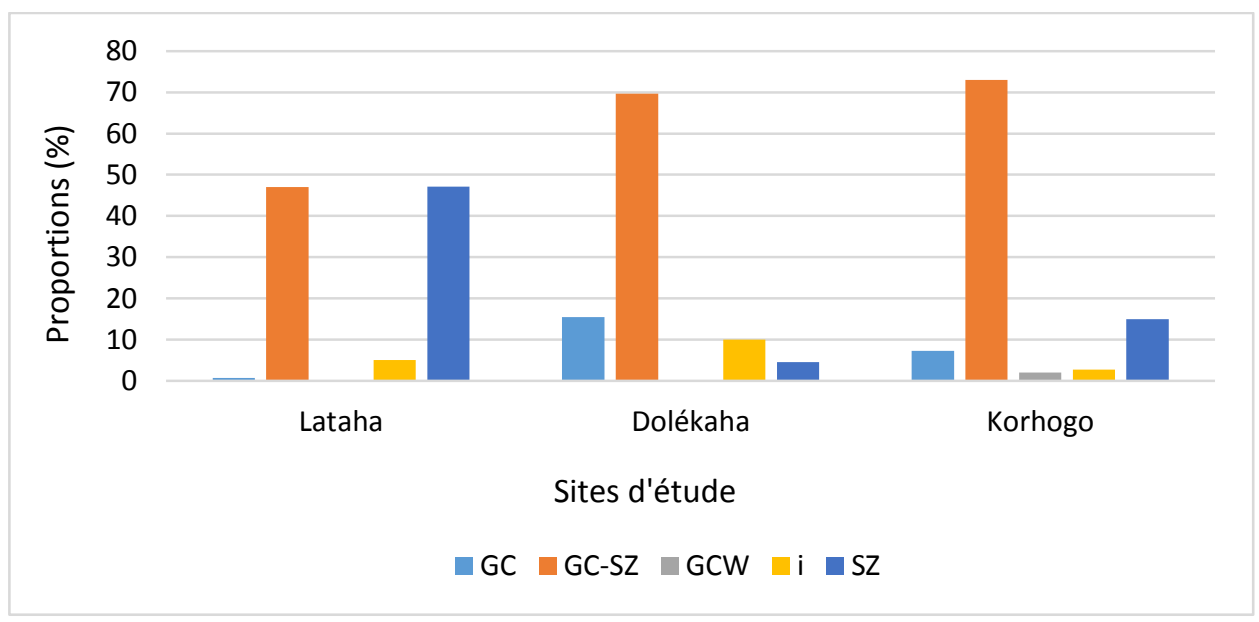

Figure 4: Proportions des types chorologiques en fonction des sites inventoriés.

Tableau 2 : Liste des espèces à statut particulier recensées dans les trois ilots de forêts.

\begin{tabular}{|c|c|c|c|c|c|c|}
\hline \multirow[t]{2}{*}{ Nom scientifique } & \multirow[t]{2}{*}{ Famille } & \multicolumn{2}{|c|}{ Statut particulier } & \multicolumn{3}{|c|}{ Sites } \\
\hline & & IUCN (2018) & $\begin{array}{c}\text { Aké-Assi } \\
\text { (1998) }\end{array}$ & Lataha & Dolékaha & Korhogo \\
\hline $\begin{array}{l}\text { Afzelia africana } \mathrm{Sm} . \\
\text { ex Pers. }\end{array}$ & Fabaceae & $\mathrm{Vu}(\mathrm{A} 1 \mathrm{~d})$ & - & 1 & 1 & 1 \\
\hline $\begin{array}{l}\text { Albizia ferruginea } \\
\text { (Guill. \& Perr.) Benth }\end{array}$ & Fabaceae & $\mathrm{Vu}(\mathrm{A} 1 \mathrm{~cd})$ & - & 1 & - & 1 \\
\hline $\begin{array}{l}\text { Detarium } \\
\text { microcarpum } \\
\text { Guill. \& Perr. }\end{array}$ & Fabaceae & $\mathrm{LC}$ & $\mathrm{Vu}$ & 1 & - & - \\
\hline $\begin{array}{l}\text { Khaya senegalensis } \\
\text { (Desv.) A.Juss. }\end{array}$ & Meliaceae & $\mathrm{Vu}(\mathrm{A} 1 \mathrm{~cd})$ & - & 1 & 1 & 1 \\
\hline $\begin{array}{l}\text { Milicia excelsa } \\
\text { (Welw.) C.C.Berg }\end{array}$ & Moraceae & NT & $\mathrm{Vu}$ & - & 1 & - \\
\hline
\end{tabular}




\begin{tabular}{|c|c|c|c|c|c|c|}
\hline $\begin{array}{l}\text { Mitragyna } \\
\text { ledermannii } \\
\text { (K.Krause.) Ridsdale }\end{array}$ & Rubiaceae & $\mathrm{Vu}(\mathrm{A} 1 \mathrm{c})$ & - & - & - & 1 \\
\hline $\begin{array}{l}\text { Nothospondias } \\
\text { staudtii }\end{array}$ & & & & & & \\
\hline Engl. & Simaroubaceae & $\mathrm{Vu}(\mathrm{B} 1+2 \mathrm{c})$ & $\mathrm{Vu}$ & - & - & 1 \\
\hline $\begin{array}{l}\text { Pavetta mollissima } \\
\text { Hutch. \& Dalziel }\end{array}$ & Rubiaceae & $\begin{array}{c}\mathrm{Vu}(\mathrm{A} 1 \mathrm{c} \\
\mathrm{B} 1+2 \mathrm{c})\end{array}$ & $\mathrm{Vu}$ & - & - & 1 \\
\hline $\begin{array}{l}\text { Pterocarpus erinaceus } \\
\text { Poir. }\end{array}$ & Fabaceae & $\mathrm{EN}(\mathrm{A} 3 \mathrm{~d})$ & - & 1 & 1 & - \\
\hline $\begin{array}{l}\text { Syzygium guineense } \\
\text { (Engl.) F.White }\end{array}$ & Myrtaceae & - & $\mathrm{Vu}$ & 1 & 1 & 1 \\
\hline $\begin{array}{l}\text { Vitellaria paradoxa } C \text {. } \\
\text { F. Gaertner }\end{array}$ & Sapotaceae & $\mathrm{Vu}(\mathrm{A} 1 \mathrm{~cd})$ & - & 1 & 1 & 1 \\
\hline $\begin{array}{l}\text { Voacanga thouarsii } \\
\text { var obtusa } \text { Roem. ex } \\
\text { Schultes }\end{array}$ & Apocynaceae & - & $\mathrm{Vu}$ & - & - & 1 \\
\hline Total & & & & 7 & 6 & 9 \\
\hline
\end{tabular}

Tableau 3 : Indice de diversité de Shannon et équitabilité de Piélou des fragments forestiers.

\begin{tabular}{llllll}
\hline Indices de diversités & Lataha & Dolékaha & Korhogo & \multicolumn{2}{l}{ Test de Kruskal-Wallis } \\
\cline { 5 - 6 } & & & & $\boldsymbol{X}^{\mathbf{2}}$ & $\boldsymbol{P}$ \\
\hline Indice de Shannon (H') & $2,36 \pm 0,58 \mathrm{a}$ & $2,29 \pm 0,26 \mathrm{a}$ & $2,47 \pm 0,28 \mathrm{~b}$ & 1,66 & 0,435 \\
\hline Equitabilité (J') & $0,79 \pm 0,15 \mathrm{a}$ & $0,84 \pm 0,07 \mathrm{~b}$ & $0,84 \pm 0,05 \mathrm{~b}$ & 6,26 & 0,043 \\
\hline Les valeurs des lignes portant des lettres différentes sont significativement différentes au seuil de $5 \%$.
\end{tabular}

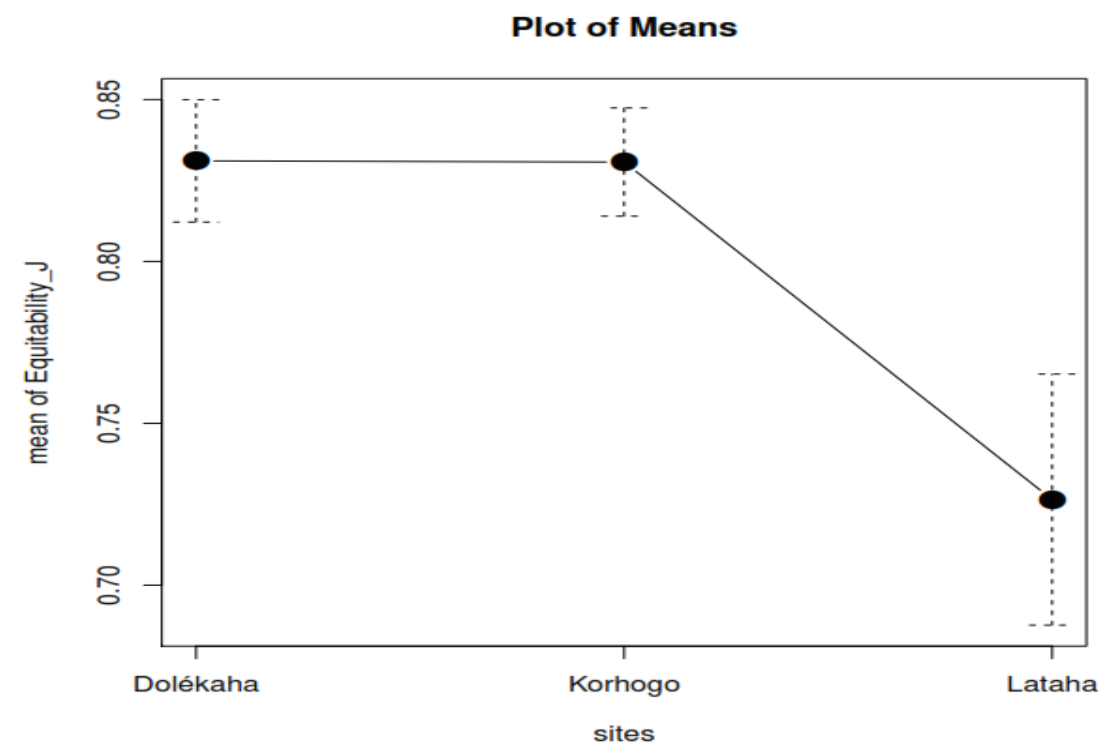

Figure 5: Représentation de la valeur des indices d'équitabilité moyenne entre les sites d'étude. 
Tableau 4 : Matrice de similarité de Sorensen entre les sites.

\begin{tabular}{llll}
\hline Sites & Lataha & Dolekaha & Korhogo \\
\hline Lataha & 1 & 0,44037 & 0,52713 \\
Dolekaha & 0,44037 & 1 & 0,5098 \\
Korhogo & 0,52713 & 0,5098 & 1 \\
\hline
\end{tabular}

\section{DISCUSSION}

\section{Richesse spécifique}

L'analyse de la richesse floristique révèle que la forêt de Lataha enregistre 74 espèces contre 49 espèces dans la forêt de Dolékaha et 65 espèces dans celle de Korhogo. La grande richesse spécifique observée sur le site de Korhogo, malgré la plus faible surface échantillonnée sur ce site pourrait provenir de son statut de forêt sacrée localisée dans la forêt classée de Korhogo. Cela montre que les effets conjugués de la sacralisation et des opérations de contrôles effectuées par la SODEFOR et les riverains contribuent fortement à la réduction de la pression anthropique sur cet îlot forestier. Ce qui a favorisé la bonne conservation et protection de la phytodiversité de cette forêt par rapport aux deux autres qui ne bénéficient que d'un seul type de gestion.

L'allure des courbes aires-espèces de ces différentes formations forestières traduit également, une augmentation de la richesse spécifique de ces forêts en fonction de la superficie échantillonnée. Cette richesse spécifique peut augmenter si l'on combine plusieurs méthodes d'inventaire botanique pouvant permettre d'obtenir des listes floristiques plus riches, même quand les superficies sont moins importantes (Vroh et al., 2010 ; Gueulou et al., 2018 ; Soro et al., 2019). Les valeurs de richesse spécifique obtenues dans cette étude sont proches ou peuvent même être supérieures à celles observées dans certaines zones humides. Nous pouvons citer les travaux de Vroh et al. (2015) dans la forêt communautaire d'Azaguié au Sud-Est de la Côte d'Ivoire (33 espèces) et ceux de Tiokeng et al. (2015) dans la forêt du Bangang à l'Ouest du Cameroun (48 et 72 espèces).
Les familles botaniques les plus dominantes au sein des îlots forestiers sont les Fabaceae, les Rubiaceae et les Apocynaceae. Cependant, les travaux de N'Guessan (2009) et Ouattara et al. (2016) tous réalisés dans les zones sèches du pays révèlent que les Poaceae constituent la famille botanique la plus abondante de la flore. Ce résultat s'explique par le fait que les relevés n'ont concerné que les ligneux. De même, plusieurs travaux effectués dans des forêts des zones humides et même sèches du pays les révèlent comme les familles botaniques les plus importantes de la flore ivoirienne (Missa et al., 2019; Yéo et al., 2017 ; Aké-Assi, 2002).

\section{Composition floristique}

Le spectre biologique de la forêt de Lataha se caractérise par une forte présence des microphanérophytes et une absence de mégaphanerophytes, à l'inverse de celle de Dolékaha qui enregistre la plus forte proportion de mégaphanerophytes et de mésophanérophytes avec de faibles proportions des microphanérophytes. L'abondance des petits arbres et l'absence de grands arbres dans la formation naturelle de Lataha se justifieraient par l'état de vielle jachère de ce site mise en défens il y a seulement 30 ans. Avant cette période, elle était sous surveillance de la population. Les activités telles que l'abattage du bois, la chasse, la récolte de produits forestiers ont entrainé la destruction de grands arbres. C'est une forêt qui se renouvelle constamment en absence de forte pression anthropique et en la faveur de sa canopée ouverte qui y laisse pénétrer la lumière. Quant à l'abondance des grands arbres et la faible proportion des petits arbres dans la forêt de Dolékaha, elle est due au caractère sacré de cette forêt où les grands 
arbres sont conservés pour les rituels d'adoration. Cette situation pourrait être également liée à des facteurs de perturbations due aux différentes pressions anthropiques exercées sur cette formation forestière. En effet, dans cette forêt sacrée protégée depuis plus de 150 ans, aucune activité d'exploitation de ressources forestière n'est autorisée aux habitants du village de Dolékaha. Cependant ce droit est réservé à la population environnante qui peut y pratiquer la chasse et prélever le bois mort pour le chauffage. Pourtant, il a été démontré que les bois morts constituent une base vitale dans la régénération naturelle de nombreuses espèces (Lachat et al., 2019). En plus, les alentours de la forêt ne sont pas épargnés des pressions pastorales. Ces différentes pressions contribuent au ralentissement du potentiel de régénération naturelle des ligneux de cette forêt. Pour le site de Korhogo, on y note une abondance des trois types biologiques (microphanérophytes, mésophanérophytes et mégaphanerophytes). Selon les études de Gueulou et al. (2018), l'abondance élevée de ces types biologiques est l'un des indicateurs de l'existence de bonnes conditions écologiques dans une forêt.

Sur le plan chorologique, les espèces de transition entre la zone guinéo-congolaise et la zone soudanaise abondent dans tous les fragments forestiers montrant ainsi une grande amplitude écologique de ces espèces dans les forêts naturelles des milieux anthropisés. Ce qui fait de ces formations forestières, des forêts denses relativement plus sèches par rapport à celle des zones humides (domaine guinéen). Selon Yéo et al. (2017) et Koulibaly (2008), qui ont travaillé dans le parc national de la Comoé, le pourcentage élevé d'espèces guinéo-congolaises et d'espèces soudaniennes indique que la région n'a pas d'affinité prononcée pour l'une ou l'autre des zones phytogéographiques. Cependant on note quelques différences entre les trois biotopes prospectés. La forte proportion des espèces soudaniennes dans la forêt de Lataha est un indicateur de fidélité de ces espèces à leur zone de confinement. La forte présence des espèces guinéennes au détriment des espèces soudaniennes dans les forêts de Dolékaha et de Korhogo serait liée au milieu toujours humide favorisant la flore guinéenne.

\section{Intérêts pour la conservation}

Le site de Korhogo regorge le plus grand nombre d'espèces à statut particulier (endémiques, vulnérables, en danger). Ce qui montre, bien le niveau élevé de la diversité floristique de ce site. Il est donc le mieux conservé. Selon Tchouto (2004) les espèces à statut particulier sont les plus sensibles aux perturbations causées par l'homme. Les activités anthropiques qui contribuent à la dégradation de la forêt, ne favorisent pas la survie de ces espèces qui recherchent un microclimat particulier.

Les proportions élevées d'espèces introduites sur le site de Dolékaha indiquent qu'il représente le site le plus perturbé. Cette situation, peut être due à une insuffisance de connaissance et un manque de formation des autorités coutumières aux pratiques d'aménagement et de protection des forêts naturelles.

\section{Diversité et similarité floristique}

Il ressort de l'analyse des indices de diversité, que la plus grande valeur d'indices de Shannon et d'équitabilité est observée à Korhogo ce qui signifie que cette forêt est encore la plus diversifiée avec une bonne répartition des individus entre les différentes espèces. En effet, la forêt de Korhogo, est une forêt ripicole. Selon les études de Sambaré et al. (2010), si les inondations prolongées constituent des freins à la phytodiversité des formations ripicoles, l'humidité des berges, les microhabitats, les microclimats et le caractère sacré sont des facteurs qui favorisent la diversité de ce site. Les mêmes observations ont été faites par Ceperley et al. (2010), alors que Dibi et al. (2008) soutiennent que les sols saturés en eau ne laissent pousser qu'un nombre réduit d'espèces adaptées à l'humidité.

Les îlots forestiers de Lataha, Korhogo et Dolékaha appartiennent à la même région floristique. Mais, les valeurs des coefficients de similitude obtenues révèlent que la forêt de 
Korhogo présente plus de similarité floristique avec celle de Lataha et de Dolékaha. Ce résultat s'explique par le fait que les sites de Lataha et de Korhogo sont caractérisés par le même type forestier et des conditions édaphiques similaires. Ce sont des galeries forestières qui partagent des espèces comme Uvaria chamae, Magaritaria discoides, Cola cordifolia, Sizizium guineense et Albizia zyzia qui ont été signalées par Sambaré et al. (2010) comme les espèces des berges sud soudaniennes du Burkina qui représente le Nord soudanien de la Côte d'Ivoire, notre zone d'étude. Quant à l'affinité floristique observée entre les sites de Korhogo et Dolékaha, elle serait due au caractère sacré de ces deux sites. Ces reliques forestières appartenant à la même zone floristique proviennent de la même flore originelle. Elles semblent résulter d'une régression du couvert forestier originel suite à des fragmentations qui n'ont laissé subsister que des forêtsreliques témoins. Elles sont généralement représentées par les forêts sacrées protégées à travers plusieurs générations. A titre indicatif, la protection et la conservation de l'espèce Antiaris africana, espèce commune à ces deux formations, est due au fait qu'elle est considérée comme le dieu protecteur de certaines forêts sacrées de la zone notamment celle de Dolékaha. Selon la population de Dolékaha, leur disparition peut entraîner de graves perturbations sociales. C'est donc à juste titre que Malan (2009) estime que la sacralisation des sites constituerait une stratégie efficace à inclure dans le processus de gestion moderne des ressources naturelles. Ce point de vue est également partagé par Fournier (2011), Koutchika et al. (2013) ainsi que Savadogo et al. (2018) pour qui, les pratiques rituelles dans les lieux sacrés ont une influence positive sur la conservation des ressources ligneuses.

\section{Conclusion}

Cette étude illustre bien la fragilité actuelle des systèmes de gestion locale des forêts par les populations et même le mode de gestion exclusif des organismes publics ou privés. Elle montre que la gestion collaborative favorise plus une bonne composition et la diversité floristique des forêts sèches. Elle participe plus à la conservation de la phytodiversité de ces forêts. En s'appuyant sur ces résultats, la cogestion devrait être de plus en plus intégrée dans les stratégies de conservation des forêts naturelles des zones sèches. Cependant pour une conservation plus efficace de ces formations naturelles, il est donc indispensable que les différents acteurs de cette gestion conjointe s'intéressent aux antécédents d'exploitations et les types d'activités qui y sont menés. Ils devraient aussi tenir compte de la durée de gestion, de la surface forestière et même des conditions écoclimatiques qui peuvent également entrainer de fortes variabilités dans la composition et la diversité floristique de ces reliques forestières.

\section{CONFLIT D'INTERETS}

Les auteurs déclarent n'avoir aucun conflit d'intérêts.

\section{CONTRIBUTIONS DES AUTEURS}

NG a assuré la collecte, le traitement des données et la rédaction du manuscrit. NDO a participé au traitement des données et la rédaction du manuscrit. $\mathrm{BC}$ et $\mathrm{AB}$ ont élaboré le protocole de recherche, dirigé et encadré cette étude; $\mathrm{AB}$ a également participé à la récolte, au dépouillement des données de terrain. $\mathrm{AKN}$ et $\mathrm{AA}$ ont contribué à l'amélioration du protocole de recherche et au choix des sites d'étude. Tous les auteurs ont contribué à la lecture et à la correction de l'article.

\section{REMERCIEMENTS}

Nos remerciements vont à l'endroit du Centre de Gestion de la Société de Développement des Forêts (SODEFOR) de Korhogo, au capitaine TAPE Bi Foua Alphonse, Directeur de ladite institution et au capitaine ABY Aka André, Chef du Service des Opérations Techniques et Commerciales de Korhogo pour leurs aides précieuses. Nous remercions, les chefs de village et populations de Dolékaha et Séhèlè qui ont toujours fait montre d'une bonne coopération. Nous 
remercions également la direction régionale du Centre National de Recherche Agronomique (CNRA) de Korhogo et Madame Djidji Hortense, Directrice de la Station de recherche de Lataha pour leur soutien logistique dans la réalisation de cette étude. Enfin, grand merci au technicien Yéo Ousmane notre guide et interprète. Les auteurs tiennent aussi à remercier les référés pour leurs remarques constructives qui ont permis d'améliorer le document.

\section{REFRERENCES}

Adou Yao YC, N'guessan KE. 2005. Diversité botanique dans le sud du parc national de Taï, Côte d'Ivoire. Afrique Science, $\quad$ 01(2): 295-313. https://www.ajol.info/index.php/afsci/art icle/viewFile/61129/49315

Aké-Assi L. 2002. Flore de la Côte d'Ivoire : catalogue systématique, biogéographie et écologie. Mémoire de Botanique systématique, Conservatoire et Jardin Botanique de Genève. Boisseria, 58: $441 \mathrm{p}$.

Aké Assi L. 2001. Flore de la Côte d'Ivoire: catalogue systématique, biogéographique et écologique. Boissiera, 57: 396. http://bibliotheques.mnhn.fr/ medias/detailstatic.aspx? INSTANCE $=$ exploitation\&RSC_BASE $=$ HORIZON\&RSCD OCID $=312431$

Aké-Assi L. 1998. Impact de l'exploitation forestière et du développement agricole sur la conservation de la diversité biologique en Côte d'Ivoire. Le Flamboyant, 48 : 20-21.

Aké-Assi L. 1984. Flore de la Côte d'Ivoire : étude descriptive et biogéographique avec quelques notes ethnobotaniques. Thèse, Université d'Abidjan, Côte d'Ivoire, $1206 \mathrm{p}$.

APG IV. 2016. An update of the Angiosperm Phylogeny Group classification for the orders and families of flowering plants. Botanical Journal of the Linnean Society, 181: 1-20. DOI: https://doi.org/10.1111/boj.12385.
Aubreville A. 1959. Flore forestière de la Côte d'Ivoire. Centre Techn. For. Trop. Nogent s/Marne, 1-3 (310): 296-286.

Bakayoko A. 2005. Influence de la fragmentation forestière sur la composition floristique et la structure végétale dans le Sud-Ouest de la Côte d'Ivoire. Thèse de Doctorat, Université de Cocody, Abidjan, 231p.

Ceperley N, Montagnini F, Natta A. 2010. Importance des sites sacrés pour la conservation des forêts galeries au centre Benin. Bois et Forêts des Tropiques, 303(1): 5-23. DOI: 10.19182/bft2010.303.a20450

Chatelain C, Ake-Assi L, Spichiger R, Gautier L. 2011. Cartes de distribution des plantes de Côte d'Ivoire. Boissiera, 64:1327.

Chape S, Blyth S, Fish L, Fox P, Spalding M. 2003. United Nations List of Protected Areas. IUCN, Gland, Switzerland and Cambridge, 44p. http://w ww.unepwcmc.org/wdpa/unlist/2003_UN_LIST.p df

Dudley N. 2008. Lignes directrices pour l'application des catégories de gestion aux aires protégées. UICN, Gland, Suisse, 96p. https://portals.iucn.org

Dibi H, Adou YC, N'guessan KE, Koné M, Sagne YC. 2008. Analyse de la diversité floristique du parc national de la Marahoué, Centre-Ouest de la Côte d'Ivoire. Afrique Science, 4(03): 552557.

Djègo J, Gibigaye M, Tente $\mathrm{B}$, Sinsin $\mathrm{B}$. 2012. Analyse écologique et structurale de la forêt communautaire de Kaodji au Bénin. Int. J. Biol. Chem. Sci., 6(2): 705713.

DOI: http://dx.doi.org/10.4314/ijbcs.v6i2.14

Ervin J. 2003. Rapid Assessment of Protected Area Management Effectiveness in Four Countries. BioScience, 53(9): 833-841. DOI: https://doi.org/10.1641/0006 3568(2003)053[0833

Felfili JM, Silva JMC, Sevilha AC, Fagg CW, Walter BMT, Nogueira PE, Rezende AV. 2004. Diversity, floristic and structural patterns of cerrado vegetation 
in Central Brasil. Plant Ecology, 175: 37-46.

https://s3.amazonaws.com/academia.edu. documents/46102368/Diversity_floristic _an _structural_patte20160531-14914psur7k.

Fournier A. 2011. Consequences of wooded shrine rituals on vegetation conservation in West Africa: a case study from the Bwaba cultural area (West Burkina Faso). Biodivers. Conserv., 20: 1895 $-1910$. https://hal.archivesouvertes.fr/hal-00542627

Gueulou N, Ouattara ND, Konan D, Gnahoré E, Missa K, Bakayoko A. 2018. Diversité floristique et structurale de la forêt galerie du Bandama dans la Réserve Scientifique de Lamto en Côte d'Ivoire. Afrique Science, 14(4): 452-439. http://www.afriquescience.net

Guillaumet JL, Adjanohoun E. 1971. La végétation de la Côte d'Ivoire. In $L e$ Milieu Naturel de Côte d'Ivoire, Avenard JM, Eldin E, Girard G, Sircoulon J, Touchebeuf P, Guillaumet JL, Adjanohoun E, Perraud A (ed). ORSTOM $\left(n^{\circ} 50\right)$ : Paris, France ; 161263.

IUCN. 2018. Red List of Threatened Species. IUCN. http://www.iucnredlist. org/.

Koulibaly AV. 2000. Caractéristique de la végétation et de la flore de la savane soudanienne de Côte d'Ivoire: cas des alentours de la ville de Korhogo. Mémoire de DEA, Université de Cocody, Abidjan, 83 p.

Koné M. 2004. Étude de la variabilité de la couverture ligneuse en milieu de savane à partir de l'imagerie satellitale haute résolution : cas du nord de la Côte d'Ivoire (région de Korhogo).Thèse de doctorat, Université de Cocody, Abidjan, $135 \mathrm{p}$.

Koulibaly A. 2008. Caractéristiques de la végétation et dynamique de la régénération sous l'influence de l'utilisation des terres, dans des mosaïques forêts-savanes, des régions du Parc National de la Comoé et de la Réserve de Lamto. Thèse de Doctorat, Université de Cocody, 150 p.
Koutchika EIR, Agbani OP, Sinsin B. 2013. Influence des perturbations anthropiques sur la biodiversité des bois sacrés du Centre Bénin. Int. J. Biol. Chem. Sci., 7(1): 306-318. DOI: http://dx.doi.org/10.4314/ijbcs.v7i1.26

Lachat T, Brang P, Bolliger M, Bollmann K, Brändli U, Bütler R, Herrmann $\mathrm{S}$, Schneider O, Wermelinger B. 2019. Bois mort en forêt : Formation, importance et conservation. Notice pour le praticien, $12 p$.

Marthews TR, Metcalfe D, Malhi Y, Phillips O, Huaraca Huasco W, Riutta T, Ruiz Jaén M, Girardin C, Urrutia R, Butt N, Cain R, Oliveras Menor I, Colleagues from the RAINFOR and GEM networks. 2012. Measuring Tropical Forest Carbon Allocation and Cycling: A RAINFORGEM Field Manual for Intensive Census Plots (v2.2). Manual of Global Ecosystems Monitoring network. http://gem.tropicalforests.ox.ac.uk/.

Malan DF. 2009. Religion traditionnelle et gestion durable des ressources floristiques en Côte d'Ivoire : le cas des Ehotilé, riverains du parc national des îles Ehotilé. Vertigo, 9(2): 1-11. DOI: 10.4000/vertigo.8661

Missa K, Soro D, Seguena F, Bakayoko A. 2019. Floristic and structural studies of the forest blocks of Nangui Abrogoua University, Cote d'Ivoire. Journal of Biodiversity and Environmental Sciences, $\quad \mathbf{1 4}(1)$ : $\quad$ 61-71. https://innspub.net

N'Guessan KE. 2009. Projet d'élaboration d'une base de données numérique sur la flore et la végétation du Parc National de la Comoé, au Nord-Est de la Côte d' Ivoire. Rapport final de projet «Centre National de Floristique », Abidjan, 37 p.

N'Guessan KA, Ahoba A, Issali AE, Akanvou R, Sékou D, Ouattara N'K, Louppe D. 2018. La haie vive, une technique durable et peu onéreuse pour protéger les cultures et terroirs ruraux. Journal of Applied Biosciences, 127: 12867-12873. DOI: https://doi.org/10.4314/jab.v127i1

Ouattara D, Kouame D, Tiebré MS, kouadio YJC, N'Guessan KE. 2016. Biodiversité 
végétale et valeur d'usage en zone soudanienne de la Côte d'Ivoire. Int. J. Biol. Chem. Sci., 10(3): 1122-1138. DOI: http://dx.doi.org/10.4314/ijbcs.v10i3.18

Ouattara N. 2001. Situation des ressources génétiques forestières de la Côte d'Ivoire (zone de savanes). Atelier sousrégional FAO/IPGRI/CIRAF sur la conservation, la gestion, l'utilisation durable et la mise en valeur des ressources génétiques forestières de la zone sahélienne (22-24 septembre 1998), Ouagadougou. http://www.fao.org/forestry/Forestry.asp.

Poisson J. 2009. Impact de la gestion participative sur l'efficacité de conservation dans les parcs nationaux des pays sous-développés. Maîtrise en biologie et en environnement de la Faculté des Sciences, Université de Sherbrooke du Québec (Canada), 121p

Raunkiaer C. 1934. The Life Forms of Plants and Stastical Plant Geography. Clarendron Press : Oxford.

Savadogo S, Sambare O, Thiombiano A. 2018. Écologie et diversité des bois sacrés et des savanes environnantes du secteur sud-soudanien du Burkina Faso (Afrique de l'Ouest).Journal of Animal \&Plant Sciences, 35(3): 5715-5733. http://www.m.elewa.org/JAPS

Sinsin B. 2001. Formes de vie et diversité spécifique des associations de forêts claires du nord du Bénin. Syst. Geogr. Pl., 71: 873-888. DOI: $10.2307 / 3668725$

SODEFOR. 2018. Plan d'aménagement de la forêt classée de Korhogo - 2019-2028. SODEFOR, 58p.

Soro Y, Ouattara ND, Missa K, Bakayoko A. 2019. Analyse de la diversité floristique de quelques îles aménagées du barrage de Buyo (Côte d'Ivoire). European Scientific, 15(18): 165-182. DOI: http://dx.doi.org/10.19044/esj.2019.v

Tchouto MGP. 2004. Plant diversity in central African rain forest. Implications for biodiversity conservation in Cameroon. $\mathrm{PhD}$ thesis, Wageningen University, Wageningen, Netherlands, 208 p.
Tiebré MS, Ouattara D, Adou Yao CY, Gnagbo A, N'Guessan KE. 2016. Caractérisation de la flore, de la végétation et potentiel de conservation de la biodiversité végétale en zone d'activités anthropiques dans le Nord-est de la Côte d'Ivoire. International Journal of Innovation and Applied Studies, $\quad$ 17(3): $\quad$ 893-900. http://www.ijias.issr-journals.org

Tiébré MS, Ouattara D, Vroh BTA, Gnagbo A, N'Guessan KE. 2016. Diversité floristique et disponibilité des plantes utilitaires en zone soudanienne de la Côte d'Ivoire. Journal of Applied Biosciences, 102: 9699-9707. http://dx.doi.org/10.4314/jab.v102i1.4

Tiokeng B, Mapongmetsem PM, Nguetsop VF, Tacham WN. 2015. Biodiversité floristique et régénération naturelle sur les Hautes Terre de Lebialem (Ouest Cameroun). Int. J. Biol. Chem. Sci., 9(1): 56-68.

DOI: http://dx.doi.org/10.4314/ijbcs.v9i1.6

Vroh BTA, Adou Yao CY, Kouame D, Kpangui KB, Gone BZB, N'Guessan KE. 2015. Trees species diversity and above ground biomass in three tropical forest types in Azaguié area, Côte d'Ivoire. Global Advanced Research Journal of Plant Science, 1(2): 030038.

http://www.garj.org/garjps/index.htm

Vroh Bi TAA, Kouame Y, N'da D, N'guessan K. 2010. Diversité floristique et structurale sur le Site d'une Réserve Naturelle Volontaire à Azaguié, Sud-est de la Côte d'Ivoire. European Journal of Scientific Research, 45(3): 411-421.

http://www.eurojournals.com/ejsr.htm

Yéo ZS, Sangne YC, Barima YSS, Bamba I, Kouakou KA. 2017. Diversité floristique et structure des fragments forestiers du Sud-Ouest du Parc National de la Comoé après les conflits des années 2000 en Côte d'Ivoire. European Scientific Journal, 13 (2): 421-439. $\quad$ DOI: 10.19044/esj.2016.v13n2p421 